\title{
WILEY-VCH
}

\section{Nanoparticle Dynamics in Oxide-Based Memristive Devices}

\author{
Stefan Tappertzhofen*, Giuliana Di Martino, Stephan Hofmann
}

Dr. Stefan Tappertzhofen

Department of Engineering, Electronic Engineering Division, University of Cambridge, CB3 0FA Cambridge, United Kingdom; now at aixACCT Systems GmbH, Talbotstrasse 25, 52068 Aachen, Germany

*E-mail: stefan.tappertzhofen@ @rwth-aachen.de

Dr. Giuliana Di Martino

NanoPhotonics Centre, Cavendish Laboratory, University of Cambridge, CB3 0HE

Cambridge, United Kingdom

Prof. Stephan Hofmann

Department of Engineering, Electronic Engineering Division, University of Cambridge, CB3 OFA Cambridge, United Kingdom;

Keywords: nanoparticles, plasmonics, memristors, redox reactions, in situ characterization

\begin{abstract}
In microelectronics a device's functionality is shaped by its interfaces. While classical semiconductor research aimed for preparation of nearly ideal interfaces, the emerging paradigm is that new functionalities can arise from interfaces with less perfection. Memristive devices rely on the control of such less-perfect interfaces. They are the building blocks for a new range of applications, including new memory and logic architectures and neuromorphic computing. Research on memristive systems and applications demands an interdisciplinary approach across disciplines including solid-state physics, electrochemistry and biochemistry. Advanced metrology is the key for a better understanding and finally a better control of such interfaces and novel device technologies. In this featured article we highlight such recent advances in characterization and the understanding of electrochemical reactions on the (sub-) nanoscale and the dynamics of the nanoparticles and clusters involved during operation of memristive devices acting as a model system. We focus particularly on in operando real time monitoring of the memristive switching effect by transmission electron microscopy and a novel plasmon enhanced spectroscopy method.
\end{abstract}




\section{WILEY-VCH}

\section{Introduction}

Silicon electronics is arguably the most understood and well-modelled technology of our times. The functionality of a device is essentially shaped by the control of its interfaces. The ambition of preparing ideal interfaces in the past obstructed one's sight that revolutionary new functionalities can arise from functional interfaces with less perfection. Rather than being siloed in one research field, understanding and exploitation of these new functionalities demand an interdisciplinary mindset across disciplines ranging from (electro-) chemistry and catalysis to materials science, solid-state physics, electrical engineering and biochemistry. Advanced metrology is the key to gain a deeper understanding and pave the way for better control of such interfaces and all of the many emergent device technologies. This topical review focuses on memristive devices, highlighting their unique model character in terms of exploration of new functional interfaces beyond classical semiconductor physics. Particularly, we highlight how these devices are ideally suited to enable in operando probing.

This review is structured in two parts. The first part introduces the current state of research on the physical and electrochemical processes involved during memristive switching. Recent advances in spectroscopic and microscopic characterization methods are highlighted in the second part. Given the range of ex situ and in in operando spectroscopic and microscopic characterization methods, a particular focus will here be on transmission electron microscopy (TEM) and a very recently established spectroscopic method based on plasmonic nanocavities.

\section{Solid-State Electrochemistry in Memristive Switches}

For detailed reviews on memristive devices with focus on the material science and device physics $^{1,2}$, electrochemistry on the (sub-) nanoscale ${ }^{3}$, modeling $^{4,5}$, and applications ${ }^{6,7}$, we refer to existing literature. For memristive switches (also termed as Resistive Switching RandomAccess Memories, RRAMs) a distinction is typically made between Conductive Bridge RAMs 


\section{WILEY-VCH}

(CBRAMs, sometimes termed as Electrochemical Metallization cells, ECM) and Valence Change Mechanism (VCM) RAMs (sometimes called Oxide-based RAM, oxRAM). ${ }^{8} 9$ The working principle of a memristive device relies on the ultra-fast ${ }^{10,11}$ and in most cases localized generation and movement of mobile ions within a nanoscale insulating material by applying an appropriate voltage between the device's two terminals. Aggregation in form of metal clusters or nanoparticles, or depletion of ions leads in the formation or rupture of electronically conductive paths between both terminals and allows for manipulation of the device resistance typically within several orders of magnitude. ${ }^{12,13}$ At least two different resistance levels (low and high resistance) are used to encode at least two different logic levels (ON and OFF state).

\subsection{Conductive Bridge RAMs}

In CBRAMs cells a nanoscale conductive metallic path between an electrochemical active working electrode/anode (such as $\mathrm{Ag}$ or $\mathrm{Cu}$ ) and a reasonably inert counter electrode/cathode (such as $\mathrm{Pt}, \mathrm{Au}$ or $\mathrm{TiN}$ ) is formed or ruptured by generation, movement and reduction of mobile metal cations (i.e. $\mathrm{Ag}^{+}$or $\mathrm{Cu}^{z+}$, where $z$ denotes the charge value of the ionic species). CBRAMs are typically based on oxides ( $\operatorname{such}$ as $\mathrm{SiO}_{2}{ }^{14}$ or $\mathrm{Al}_{2} \mathrm{O}_{3}{ }^{15}$ ), higher chalcogenides ${ }^{16}$ (e.g. GeSe), classical solid-state cation conductors (e.g. $\mathrm{AgI}^{17}$ ) and polymers (such as $\mathrm{PVDF}^{18}$ or $\mathrm{PEO}^{19}$ ). In the simplest case, anodic oxidation of a metal electrode Me generating cations $\mathrm{Me}^{z+}$ takes places when a positive voltage is applied between the anode and cathode (anodic oxidation): ${ }^{8}$

$$
\mathrm{Me} \rightarrow \mathrm{Me}^{z+}+z e^{-}
$$

The counter reaction is the reduction of cations at the cathode and obeys

$$
\mathrm{Me}^{z+} \rightarrow \mathrm{Me}-z e^{-}
$$

Equation (1) and (2) may explain resistive switching in a classical ion conductor such as $\mathrm{AgI}$ which consists of a matrix of sufficiently mobile cations in the pristine state but cannot explain the injection of cations in cation-free insulators like $\mathrm{SiO}_{2}$ or $\mathrm{GeSe}$. In these systems, charge neutrality is not fulfilled for equation (1) since equation (2) is inhibited due to the lack of 


\section{WILEY-VCH}

reducible cations close to the cathode (at least in the pristine state). In contrast, equation (3): ${ }^{20-}$ 22

$$
\mathrm{O}_{2}+2 \mathrm{H}_{2} \mathrm{O}+4 e^{-} \rightarrow 4 \mathrm{OH}^{-}
$$

has been suggested as the required counter reaction at the cathode to fulfill charge neutrality before cations agglomerate at the cathode for cation reduction (equation (2)). In fact, a number of studies have demonstrated the relevance of hydroxyl ions $\mathrm{OH}^{-}$being present both for resistive switching.

In a simplified model, cations drift from the anode towards the cathode where they get reduced when a positive voltage is applied between anode and cathode. ${ }^{8}$ Reduction of cations results in a local enhancement of the electric field, and consequently, a metal filament grows from the cathode towards the anode. However, the movement and redox-reactions of cations as well as the formation and rupture of metallic inclusions into the insulator (i.e. filament and nanoparticles and -clusters) are in general subject to a complex interplay of the local ion concentration $^{23}$, ion mobility ${ }^{24,25}$, redox rate $^{26}$ as well as local nucleation and insulator morphology ${ }^{27}$. Details will be discussed in section 3 .

\subsection{Valence Change Mechanism}

Transition metal oxides like $\mathrm{ZrO}_{2}, \mathrm{SrTiO}_{3}$ or $\mathrm{Ta}_{2} \mathrm{O}_{5}$ are the choice of materials for $\mathrm{VCM}$ cells. In VCM cells mobile oxygen vacancies $\mathrm{V}_{\mathrm{O}}{ }^{\bullet}$ are responsible for the formation of an oxygen deficient and electronically conductive path ("plug") between a low (e.g. Zr, Ti, Hf, Ta) and a high (e.g. Pt) work function electrode during an initial electro-forming step. ${ }^{1}$ In thermodynamic equilibrium the oxygen vacancy concentration in a transition metal oxide obeys in Kröger and Vink notation

$$
2 \mathrm{O}_{\mathrm{O}} \leftrightarrow \mathrm{O}_{2}(\mathrm{~g})+2 \mathrm{~V}_{\mathrm{O}} \cdot{ }^{\prime}+4 \mathrm{e}^{\prime}
$$

where $\mathrm{O}_{\mathrm{O}}$ are oxygen ions on regular lattice sites and $\mathrm{O}_{2}(\mathrm{~g})$ is oxygen in the ambient gas phase. Hence, the oxygen vacancy concentration is controlled by the ambient partial pressure of 


\section{WILEY-VCH}

oxygen. According to the current understanding, the concentration of oxygen vacancies between the plug's tip and the high work function electrode modulates a Schottky barrier effectively making the device more or less electronically conductive. ${ }^{1}$ Theoretical models predict a strong increase of the oxygen vacancy mobility due to very localized Joule heating during resistive switching and electric field enhancement. ${ }^{28}$ The general validity of models for VCM cells is currently under debate as recent studies show that in some VCM systems mobile cations may significantly contribute to the switching effect. ${ }^{29,30}$

In addition to resistive switching, many transition metal oxides are also ferroelectric. A very promising material is Hafnium oxide. ${ }^{31-33}$ Both ferroelectric ${ }^{34}$ and resistive switching has been reported in $\mathrm{HfO}_{\mathrm{x}}$ and both effects appear to be influenced by oxygen vacancies. While resistive switching is typically observed in amorphous $\mathrm{HfO}_{x}$, and ferroelectric switching is reported for crystalline $\mathrm{HfO}_{\mathrm{x}}$, a recent study demonstrated a reversible combination of resistive and ferroelectric switching in a single poly-crystalline Sr-doped cell. ${ }^{35}$

When replacing the metal-insulator-metal structure of classical VCM cells by flexible nanocomposites and colloidal suspensions, based on e.g. $\mathrm{ZnO}$, resistive switching becomes more complex. These switches have been recently categorized as interfacial coupling mechanism (ICM) devices ${ }^{36}$ : Experimental results indicate here that in those devices local metal adatom doping of $\mathrm{ZnO}$ nanowires is important for conductance changing even for low metal concentrations.

\section{Recent Advances in Characterization Methods of Memristive Devices}

Only little is understood about the actual working principle of memristive switches. In case of CBRAMs, ion mobility derived from studies based on bulk oxides cannot explain resistive switching within micro- to nanoseconds and in contrast to VCM cells Joule heating can be excluded in most cases. For VCM cells the model of a modulated Schottky barrier is still part of ongoing research. In both systems, the morphology and processes for formation, rupture and 


\section{WILEY-VCH}

growth of filaments is not well understood (including the filament growth direction in CBRAMs). Recent studies suggest a prominent role of locally separated nanoparticles and clusters instead of fully continuous filaments. ${ }^{26,27}$ In addition, in both systems the signature of quantized conductance effects can be observed at room temperature ${ }^{37,38}$ indicating the importance of atomic processes during the resistive switching effect.

This section focusses on the advances in a number of widely used characterization methods, namely voltage-sweeps and pulse measurements, electrochemical cyclic voltammetry, and $e x$ situ and in operando electron microscopy. Finally, a novel non-destructive in operando spectroscopic technique is introduced based on plasmonic cavities.

\subsection{Voltage-Sweeps and Fast Pulse Measurements}

The most common methods to study resistive switching are quasi-static voltage-sweeps, which show a hysteresis in the voltage-current characteristics due to the ON and OFF state device resistance, and (fast) pulse measurements, where a voltage pulse is applied with variable amplitude and length. An example of a quasi-static voltage sweep with the current plotted in logarithmic scale is shown in Figure 1a for a $\mathrm{Ta}_{2} \mathrm{O}_{5}$ based VCM device. ${ }^{10}$ To prevent damage of the device by high currents in the ON state a current compliance is used in many experiments. It should be noted, however, that the current compliance is actively regulated by the external source meter and the regulation speed can be much longer than the switching speed of the device (e.g. up to hundreds to ms compared to some tens of nanoseconds). This may lead to artefacts in the switching behavior that have been reported for example for VCM cells. ${ }^{39}$

Pulse measurements do not allow a characterization of the full voltage-current behavior of the device, but are used for measurement of the switching speed and kinetics and are much closer to the actual operation conditions of a practical memory device. Most resistive switches have demonstrated non-linear switching characteristics with switching times between several ms to ns depending on the applied pulse voltage. ${ }^{28,40}$ By plotting the slope of the switching speed vs. 


\section{WILEY-VCH}

voltage amplitude (Figure 1b) different limiting regimes (namely nucleation limitation, electron transfer limitation and drift or mixed limitation regimes) can be identified. ${ }^{11}$
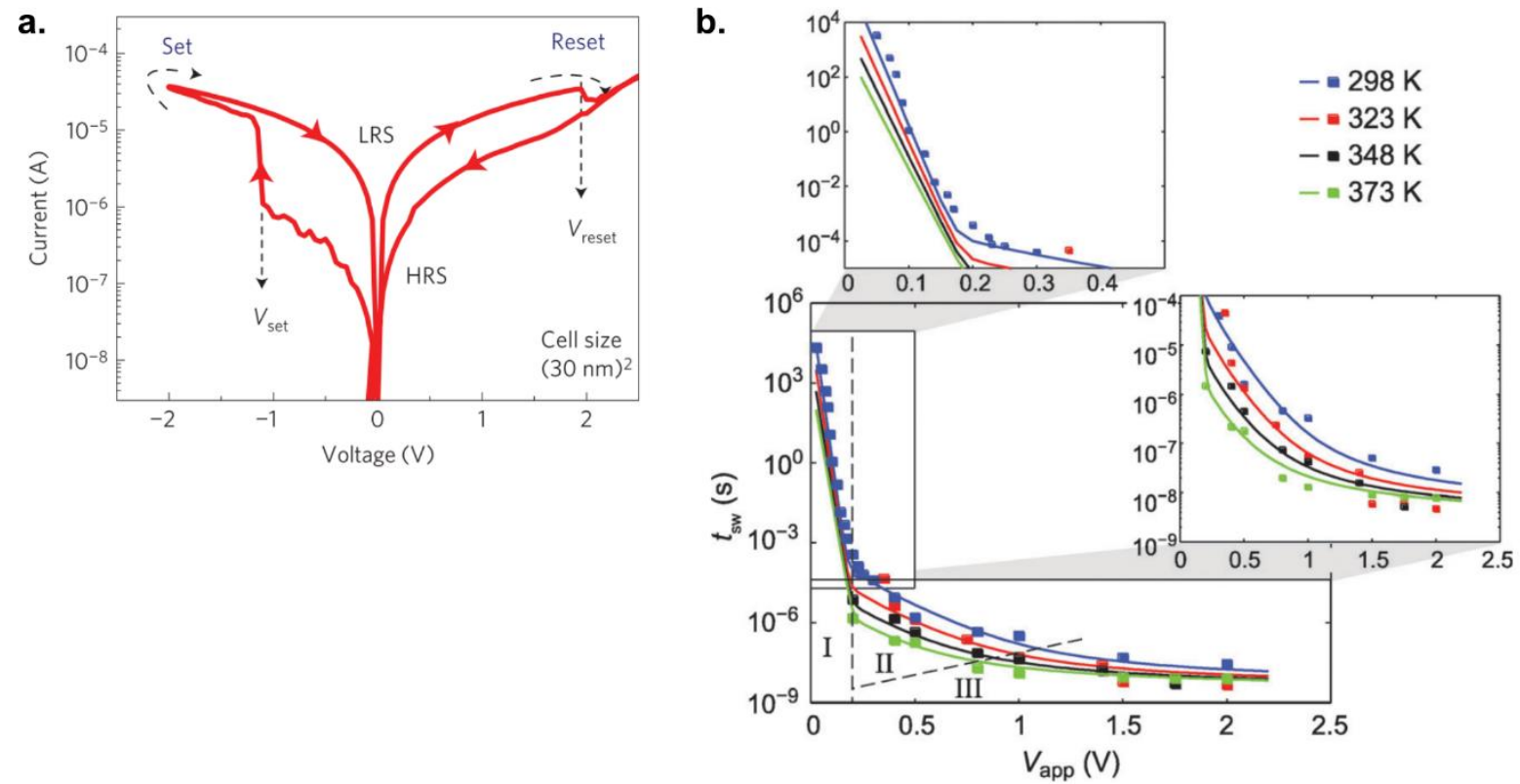

Figure 1: Electrical characterization of memristive switches. a) Quasi-static current-voltage sweeps (redrawn with permission from Ref. ${ }^{10}$ published by Springer Nature), b) non-linear switching kinetics (switching speed $t_{\mathrm{sw}}$ vs. switching/SET voltage $V_{\mathrm{app}}$ ) - redrawn with permission from Ref. ${ }^{11}$ published by the PCCP Owner Societies.

\subsection{Electrochemical Cyclic Voltammetry}

For cyclic voltammetry (CV) a typically bipolar voltage sweep is applied between the active and the counter electrode while simultaneously monitoring the current. The recorded current is plotted versus the voltage potential in respect to a reference electrode and is affected by the scan rate (i.e. the voltage sweep rate) and the signature of redox reactions that occur during the voltage sweep. CV is a widely used method in electrochemistry but its application is limited for thin films where the use of a non-polarizable reference electrode within the solid electrolyte is challenging. ${ }^{41}$ Nevertheless, cyclic voltammetry can be used in a two-terminal configuration to reveal qualitative information of the redox processes involved in resistive switches. ${ }^{23} \mathrm{CV}$ is 
very similar to the voltage-sweep method but the voltage amplitude is limited so that a complete resistance transition is suppressed.
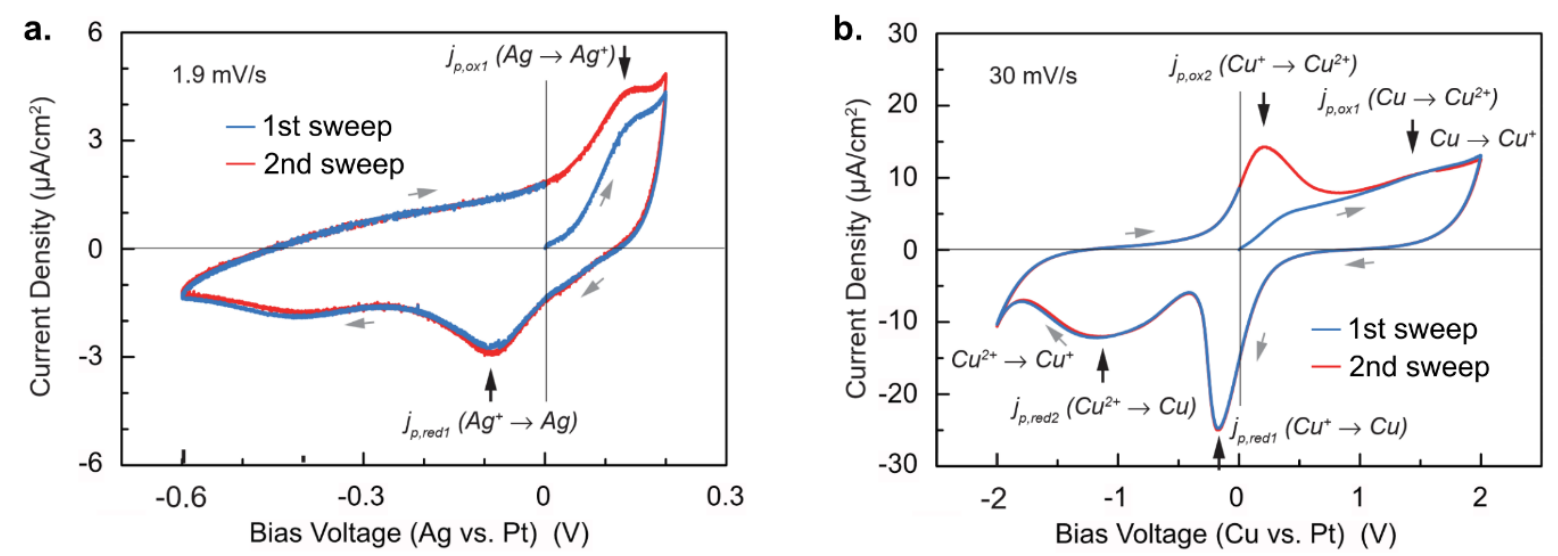

Figure 2: Cyclic voltammetry for a (a) silver- and (b) copper-based CBRAM (i.e. $\mathrm{Me} / \mathrm{Ta}_{2} \mathrm{O}_{5} / \mathrm{Pt}$, with $\mathrm{Me}=\mathrm{Ag}, \mathrm{Cu})$. Redrawn with permission Ref. ${ }^{25}$ published by WILEY - VCH Verlag $\mathrm{GmbH}$.

Figure 2a depicts $\mathrm{CV}$ curves for a $\mathrm{Ag} / \mathrm{Ta}_{2} \mathrm{O}_{5} / \mathrm{Pt} \mathrm{ECM}$ cell. At the beginning, the insulator is nearly free from mobile cations and the current starts in the origin. The current increases by increase of the applied voltage indicating the formation of $\mathrm{Ag}^{+}$cations. Once the voltage excesses the point where further anodic oxidation is limited by the diffusion of cations into the insulator a current peak is recorded. When sweeping back from the vertex potential a second current peak is observed indicating the diffusion limited reduction of $\mathrm{Ag}^{+}$. While no mobile cations are found in the pristine state (hence, the current starts in the origin), in this purely dynamic electrochemical system not all cations may be reduced in the subsequent cycles, and the current does not start at the origin anymore. In contrast to $\mathrm{Ag}, \mathrm{Cu}$ ions can be onefold $\mathrm{Cu}^{+}$ or twofold $\mathrm{Cu}^{2+}$ charged and oxidation and reduction between these species can take place:

$$
\begin{aligned}
& \mathrm{Cu}^{+} \rightarrow \mathrm{Cu}^{2+}+\mathrm{e}^{-} \\
& \mathrm{Cu}^{2+} \rightarrow \mathrm{Cu}^{+}-\mathrm{e}^{-}
\end{aligned}
$$




\section{WILEY-VCH}

By considering the standard redox potentials for $\mathrm{Cu}$ one can qualitatively link the current peaks in Figure $\mathbf{2 b}$ to the respective redox reaction. ${ }^{24,25}$

With the current density (given by the device geometry) and integration of the current peaks one can also qualitatively estimate the ion concentration and ion mobility. For ECM cells the ion concentration was found to be of the order of some $\mathrm{mmol} / \mathrm{cm}^{3}$ and the mobility is of the order of $10^{-12} \mathrm{~cm}^{2} / \mathrm{Vs}^{23}$, which is orders of magnitude higher than what can be estimated from bulk data. It has been observed that the ion concentration decreases as the sweep rate increases indicating that for high sweep rates less time is available for oxidation. ${ }^{24}$ Interestingly, the ion mobility is also affected by the sweep rate, and hence, ion concentration. The mobility decreases with increasing ion mobility, and thus, ion-ion interactions cannot be neglected in these systems. The current response, and with that, the ion concentration and ion mobility are also affected by the ambient conditions. In particular, the partial pressure of water and oxygen are important factors for the ionic current as well as the catalytic activity of the cathode for water and oxygen reactions enabling the required counter reactions to keep charge neutrality. ${ }^{21,22}$
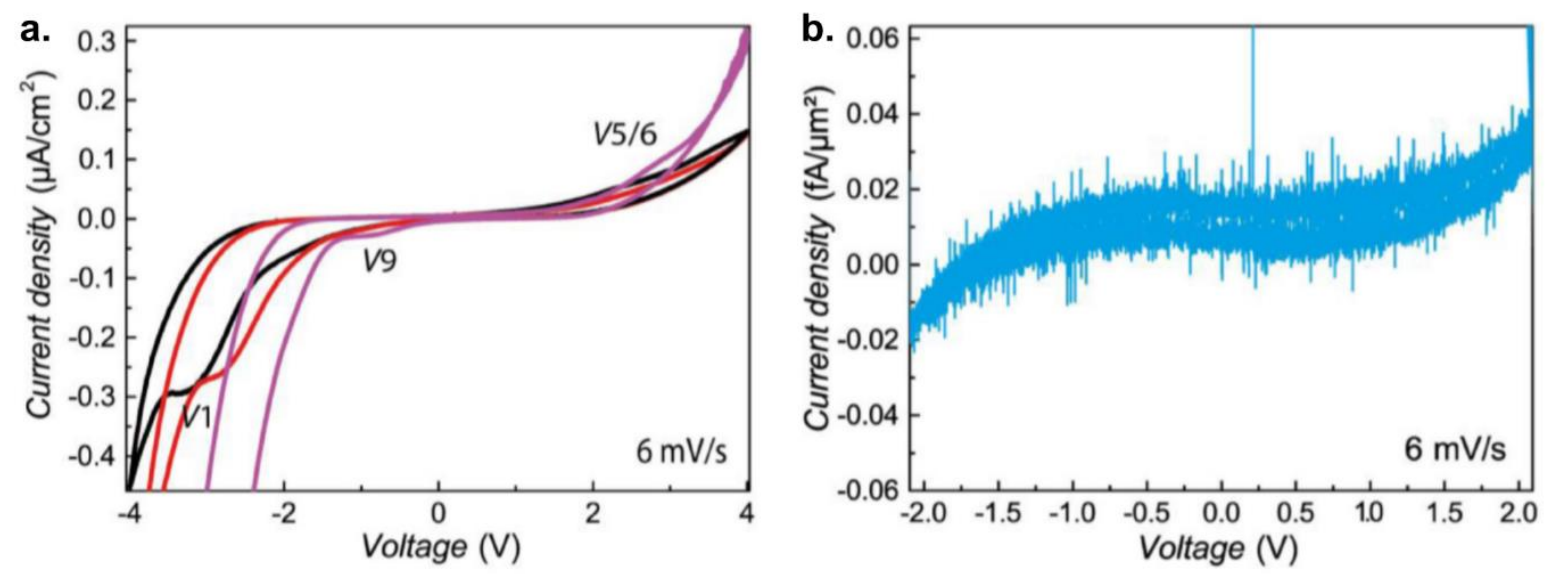

Figure 3: Cyclic voltammetry for a $\mathrm{Ta} / \mathrm{Ta}_{2} \mathrm{O}_{5} / \mathrm{Pt} \mathrm{VCM}$ cell (a) in moisture and (b) in vacuum. Reprinted with permission from Ref. ${ }^{42}$ published by WILEY - VCH Verlag GmbH.

Similar CV behavior can be observed in VCM cells supporting the assumption that VCM switching is also related to redox reactions (Figure 3). However, the interpretation of the CV 


\section{WILEY-VCH}

curves appears to be far more challenging since partial oxygen-related redox reaction as well as the effect of redox reactions where ambient oxygen and water are involved are superposed (compare Figure 3a when moisture is present and Figure 3b in vacuum). ${ }^{30,42}$ Moreover, recent studies suggest that especially close to room temperature some of the cations from the transition metal oxide lattice contribute to the ionic current, and may thus, affect the CV curves as well. ${ }^{29}$ Cyclic voltammetry is a powerful tool for analysis of mass transport and redox processes before the actual memristive switching takes place. However, CV does not provide sufficient information on the localized switching effect itself nor does it allow to gain insight into the morphology and geometry of the formed filaments.

\subsection{Transmission Electron Microscopy}

Transmission electron microscopy allows for high-resolution imaging of the resistive switching effect. Using ex situ TEM, filaments and metal clusters as well as the (change of the) morphology of the switching material before and after switching can be studied down to the mesoscopic scale. A number of studies have demonstrated the formation and rupture of conductive paths in the ON and OFF state both for VCM and CBRAM devices. ${ }^{43-45}$ In a very recent in operando TEM study ion migration in metalo-polymer hybrid resistive switching devices have been observed combined with nanoparticle reshaping at reasonably high electric fields. ${ }^{46}$ TEM imaging on CBRAMs is typically easier due to the good material contrast compared to VCM cells. In CBRAMs, the filament is usually made of different atoms (e.g. Ag or $\mathrm{Cu}$ ) compared to the switching material, while in VCM devices, the conductive path is typically a sub-stoichiometric or nearly metallic (oxygen-deficient) phase of the surrounding host material. Nevertheless, some studies have imaged conductive phases in VCM cells. For

example, in Ref. ${ }^{47}$ Kwon et al. have demonstrated the formation (Figure 4a) and rupture (Figure 4b) of a sub-stoichiometric and conductive Magnéli phase in $\mathrm{TiO}_{2}$ with a diameter as low as $10 \mathrm{~nm}$. Similar geometries for filaments have been observed for CBRAMs. 
a.

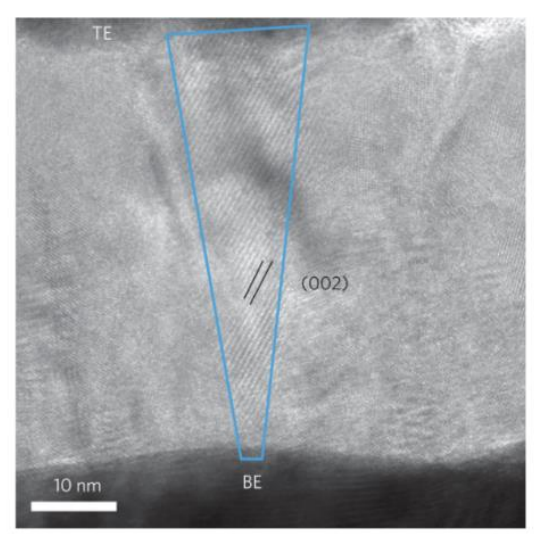

b.

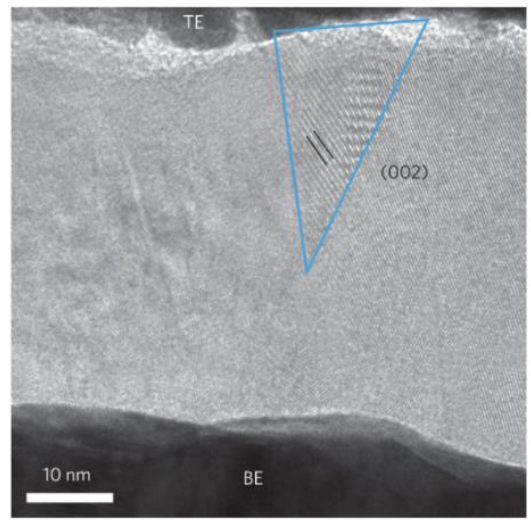

Figure 4: TEM image of a $\mathrm{TiO}_{2}$ memristive device a) after switching in the $\mathrm{ON}$ state, and b) in the OFF state. Redrawn with permission from Ref. ${ }^{47}$ published by Springer Nature.

Using in operando TEM the dynamics of nanoparticles in ECM cells have been studied in detail. Yang et al. embedded metal particles in thin oxides sandwiched between two electrodes and applied a voltage bias while monitoring the behavior of the nanoparticles. ${ }^{26}$ Figure 5a-f shows exemplarily the drift of embedded $\mathrm{Cu}$ nanoparticles. The authors report that $\mathrm{Ag}$ and $\mathrm{Cu}$ particles drift from the anode to the cathode mainly in a step-wise manner that can be explained by electrochemical bipolar electrodes ${ }^{48}$. The particle surface facing the anode locally behaves like a cathode and allows for reduction, while the opposing surface behaves like an anode and allows for oxidation. In contrast, when Pt particles are embedded larger clusters are formed that are found never to be dissolved again. $\mathrm{Ni}$ is reported to grow dendrites from the anode towards the cathode. 


\section{WILEY-VCH}

$\mathrm{Cu}$

a. A
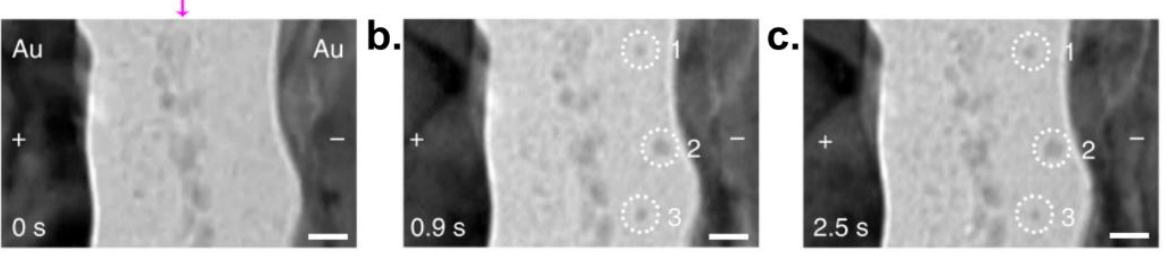

d.
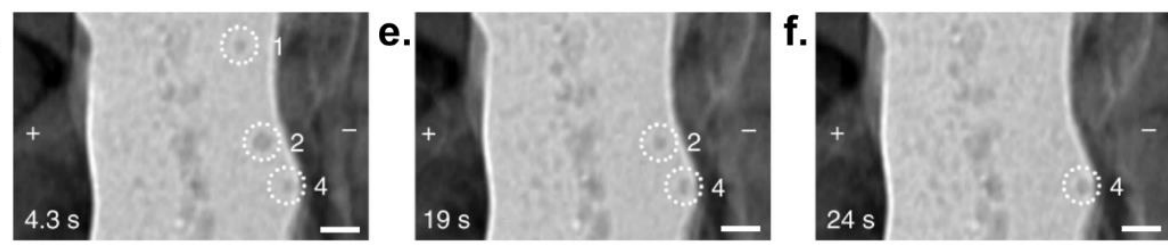

g.

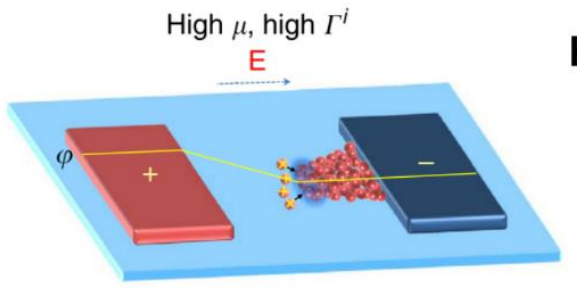

h.

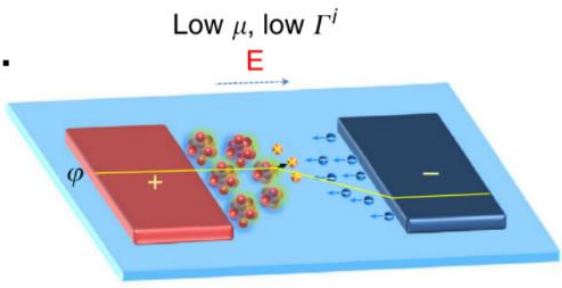

i.

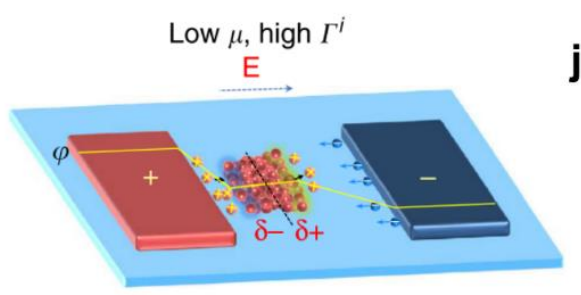

j.

High $\mu$, low $\Gamma^{i}$ $\mathrm{E}$

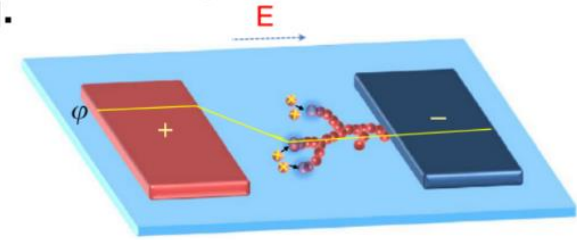

Figure 5: Dynamics of embedded nanoparticles in memristively switching oxides (here $\mathrm{SiO}_{2}$ ). a) - f) time resolved drift of $\mathrm{Cu}$ nanoparticles (1) - (3) towards a $\mathrm{Au}$ cathode monitored by in operando TEM. g) - h) schematic of the model explaining the drift and nucleation mechanism of nanoparticles as a function of the (local) ion mobility $\mu$ and redox rate $\Gamma$. Redrawn with permission from Ref. ${ }^{26}$ published by Springer Nature.

The authors explain this behavior by a complex interplay of the (local) redox and nucleation rate, and ion concentration and mobility. When both the ion mobility and redox rate are high (Figure 5g), ions can penetrate deep into the oxide and can get reduced at nearly any position at the cathode. This results in the formation of rather extended clusters growing from the cathode towards the anode. With a low ion mobility, ions can only drift a short distance from the anode and form isolated particles when the redox rate is low (i.e. reduction can only take place at specific energetically favorable positions) as shown in Figure $\mathbf{5 h}$, or an extended 


\section{WILEY-VCH}

cluster is formed in the oxide when the redox rate is high (Figure 5i). If the ion mobility is high and the redox rate is low, filaments grown from the cathode towards the anode are formed, since ions can drift deep into the oxide but are reduced only at energetically favorable positions (Figure 5j). This model helped to understand the different growth characteristics of filaments and particles in CBRAMs. Lately, the model has been extended by the observation that this interplay can dynamically change from cycle to cycle and is strongly linked to the local morphology of the switching material. ${ }^{27}$

\subsection{Plasmon enhanced Spectroscopy}

TEM is a powerful tool to image the formation and rupture of filaments in resistive switches. However, the operation conditions and samples differ in general very much from resistive switches for practical applications. Focused-ion-beam (FIB) cuts used to display cross-sectional views of devices is a destructive technique that does not allow the device to be operated afterwards. ${ }^{49}$ Additionally, Gallium contaminations cannot be avoided. For in operando TEM, devices are typically prepared on electron-transparent silicon nitride windows. In this case, imaging of filaments is non-destructive but the (lateral) device geometries are different from the (vertical) geometry of a practical device used in a memory array. This may result in a different switching material morphology and local mechanical stress. Also, the electrons used for imaging can perturb the drift of the charged ions.

Recently, a new non-destructive spectroscopic technique has been introduced, which exploits the interactions of photons with matter in the switching material..$^{50,51}$ By designing one of the two electrodes as a metallic nanodot, a strong field enhancement can be localized within the switching material. As a result, the electrodes of a memristive device behave like two metallic spheres separated by a tiny gap. The electric charges in this system can be set into oscillation by the electric field of the incident wave. Once this oscillatory motion ("plasmons") takes place, accelerated charges radiate electromagnetic energy in all directions. This secondary radiation 


\section{WILEY-VCH}

is called scattering. ${ }^{52}$ The way how the system scatters light outside the medium depends on its composition and geometry. ${ }^{53-58}$ For a system where the plasmonic field is localized in the switching material, the formation, change and rupture of conductive matter inside the switching device changes the plasma frequency which eventually results in a change of the scattered optical spectrum.

a.

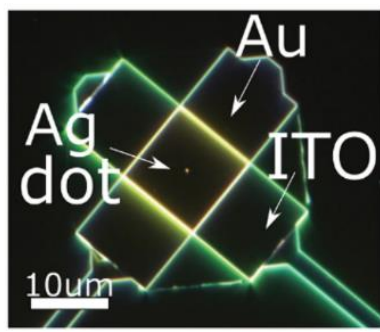

b.

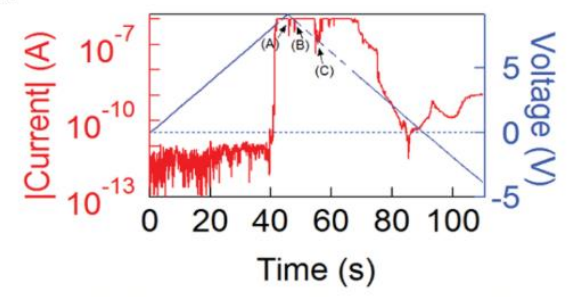

c.

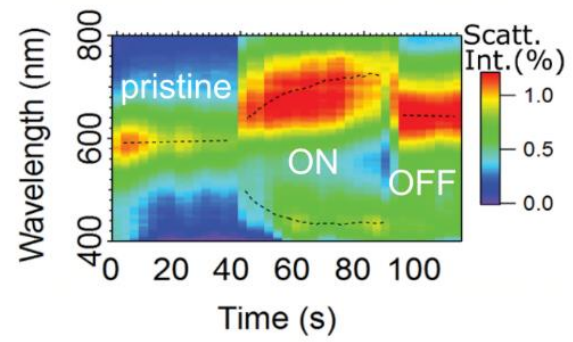

Figure 6: Nanoplasmonic enhanced spectroscopy. a) Optical microscopy image of the device under test (100 nm x $100 \mathrm{~nm} \mathrm{Ag-dot).} \mathrm{ITO} \mathrm{is} \mathrm{used} \mathrm{to} \mathrm{electrical} \mathrm{biasing} \mathrm{using} \mathrm{probe} \mathrm{needles.} \mathrm{b)}$ Transient current in response to an applied triangular voltage, and c) corresponding scattered spectra. Redrawn with permission from Ref. ${ }^{50}$ published by WILEY-VCH Verlag GmbH.

In Ref. ${ }^{50}$ the authors probed resistive switching of a Ag-nanodot/SiO $/ \mathrm{Au}$ device (electrode area $100 \mathrm{~nm}$ x $100 \mathrm{~nm}$ ) using plasmon enhanced spectroscopy. A transparent indium tin oxide (ITO) layer was used for electrical contact of the Ag-nanodot (acting as anode) and the top electrode probe needle. Figure 6a shows a dark field microscopy image of the device. The scattered spectra during device operation (Figure 6b) are shown in Figure 6c. In the pristine 


\section{WILEY-VCH}

state (i.e. device after fabrication), a peak at a wavelength around $580 \mathrm{~nm}$ is observed. Upon switching, the peak continuously shifts to larger wavelengths indicating the formation and growth of a metal filament in the oxide. When the device is switched off, the peak again moves back to shorter wavelength around $670 \mathrm{~nm}$ indicating that some leftover material remains in the oxide during OFF state. Interpretation of the spectra is non-trivial and part of ongoing research. However, the advantage of this technique is that (vertical) device geometries identical to those used in memory arrays can be studied over multiple switching cycles, in ambient conditions and without perturbing the movement of ions. Additionally, optical spectroscopy in general allows for ultra-fast probing of the switching.

a.

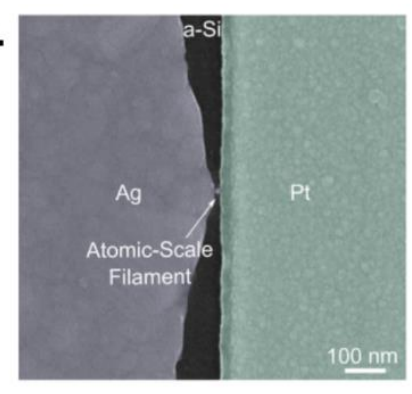

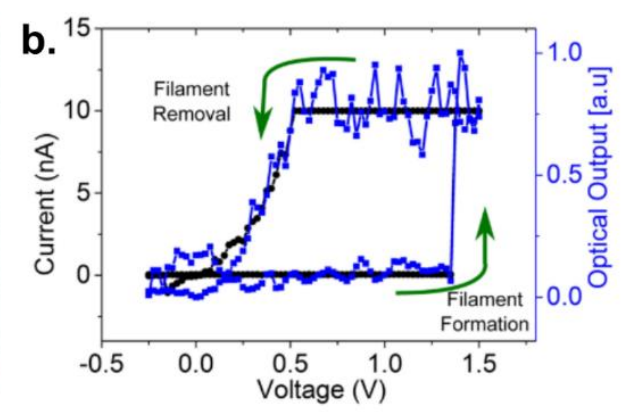

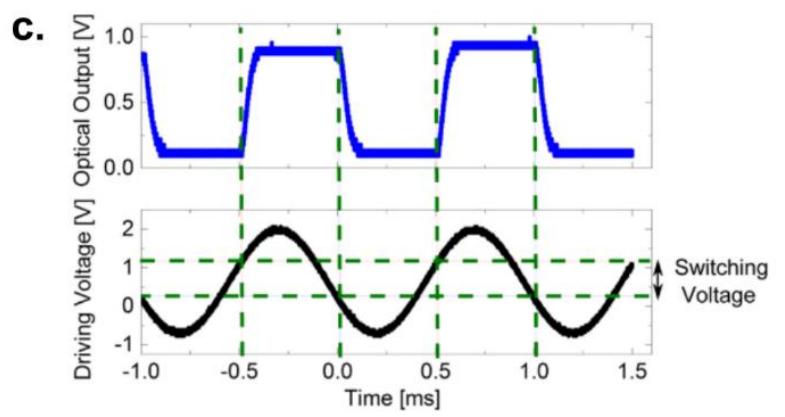

Figure 7: Optical switching with a memristive plasmonic switch. a) false-color scanning electron microscopy image of a switching device with an atomic-scale filament. b) Quasi-static current-voltage behavior and corresponding optical output. c) Modulation of an optical signal by programing the memristive device using a function generator. Redrawn with permission from Ref. ${ }^{59}$ published by the American Chemical Society. 


\section{WILEY-VCH}

The same physical processes can be also used for a new range of applications. For example, Emboras et al. fabricated an atomic-scale plasmonic switch (Figure 7a) that can be used to modulate optical signals by ultra-fast programming of the memristive device. ${ }^{60}$ Here, the optical signal is directly linked to the switching hysteresis. The authors could show optical modulation between a few $\mathrm{kHz}$ (Figure 7c) up to $1 \mathrm{MHz}$ with no more than $12.5 \mathrm{fJ} / \mathrm{bit}$ (Figure 7b) required to switch the device. ${ }^{59}$ Further improvement of the technique allowed to increase the bandwidth up to $0.5 \mathrm{Gbit} / \mathrm{s} .{ }^{61}$ Such a low energy consumption (at high speeds) for optical modulation is three orders of magnitude better than conventional $\mathrm{LiNbO}_{3}$ based Mach-Zehnder interferometer modulators, which typically operate at $10 \mathrm{pJ} / \mathrm{bit}^{62}$ However, for computing based on optical switching the bandwidth needs to be increased significantly (above some $50 \mathrm{~Gb} / \mathrm{s}^{63}$ ) to compete for example against CMOS-based advanced hybrid electro-optical microprocessors. Such high bandwidths may be supported by fast switching down to ns or below. Nevertheless, the ultralow energy consumption may open up the field for a completely new range of applications such as on-chip-level memristor based electro-optical data transmission.

\section{Conclusions}

Memristive devices are model systems that show how advanced metrology combined with an interdisciplinary mindset allow us to develop a new framework of understanding for controlling functionality in less-perfect materials and interfaces. In this review we highlighted recent developments in the characterization of memristive devices with particular attention paid to the dynamics of filaments, nanoparticles and clusters in the switching material. Advances in voltage/current-based techniques such as cyclic voltammetry were summarized. The technique allows for identification of electrochemical reactions underlying the resistive switching process. Interestingly, $\mathrm{CV}$ indicates that cations are involved in the switching both in CBRAMs and VCM-type devices. Direct observation of the growth and rupture of filaments and the dynamics of nanoparticles can be done by in operando TEM. A complex and dynamic interplay of the 


\section{WILEY-VCH}

redox and nucleation rate, and the ion concentration and mobility and the morphology of the switching material is responsible for the different geometries and growth directions of filaments reported in literature. The drawbacks of electron-based imaging in TEM can be overcome by novel plasmon enhanced spectroscopy techniques, allowing non-destructive exploration of the memristive switching process in real-time with realistic device geometries. At the same time, the underlying physical processes can be exploited for a new range of energy-efficient electrooptical applications based on ultra-fast programmable memristive devices.

\section{Acknowledgements}

S. T. acknowledges support from DFG research fellowship under grant TA 1122/1-1. G.D. acknowledges support from the Winton Program for the Physics of Sustainability and funding from ERC grant LINASS 320503, Funder Id: http://dx.doi.org/10.13039/501100000781 and EPSRC grant EP/L027151/1, Funder Id: http://dx.doi. org/10.13039/501100000266. S. T. and S. H. acknowledges support from ERC grant InsituNANO 279342, Funder Id: http://dx.doi.org/10.13039/501100000781.

\section{References}

(1) Waser, R.; Dittmann, R.; Staikov, G.; Szot, K. Redox-Based Resistive Switching Memories - Nanoionic Mechanisms, Prospects, and Challenges. Adv. Mater. 2009, 21, $2632-2663$.

(2) Sawa, A. Resistive Switching in Transition Metal Oxides. Mater. Today 2008, 11, 2836.

(3) Valov, I. Redox-Based Resistive Switching Memories (ReRAMs): Electrochemical Systems at the Atomic Scale. ChemElectroChem 2014, 1, 26-36. 


\section{WILEY-VCH}

(4) Yang, Y.; Lu, W. Nanoscale Resistive Switching Devices: Mechanisms and Modeling. Nanoscale 2013, 5, 10076.

(5) Linn, E.; Menzel, S.; Rosezin, R.; Böttger, U.; Bruchhaus, R.; Waser, R.

Nanoelectronic Device Applications Handbook: Modeling of Complementary Resistive Switches; Iniewski, J. E. M. K., Ed.; CRC Press, 2013.

(6) Zidan, M. A.; Strachan, J. P.; Lu, W. D. The Future of Electronics Based on Memristive Systems. Nat. Electron. 2018, 1, 22-29.

(7) Jeong, D. S.; Kim, K. M.; Kim, S.; Choi, B. J.; Hwang, C. S. Memristors for EnergyEfficient New Computing Paradigms. Adv. Electron. Mater. 2016, 2, 1600090.

(8) Valov, I.; Waser, R.; Jameson, J. R.; Kozicki, M. N. Electrochemical Metallization Memories-Fundamentals, Applications, Prospects. Nanotechnology 2011, 22, 289502.

(9) Waser, R.; Aono, M. Nanoionics-Based Resistive Switching Memories. Nat. Mater. 2007, 6, 833-840.

(10) Lee, M.-J.; Lee, C. B.; Lee, D.; Lee, S. R.; Chang, M.; Hur, J. H.; Kim, Y.-B.; Kim, C.J.; Seo, D. H.; Seo, S.; et al. A Fast, High-Endurance and Scalable Non-Volatile Memory Device Made from Asymmetric Ta2O(5-x)/TaO(2-x) Bilayer Structures. Nat. Mater. 2011, 10, 625-630.

(11) Menzel, S.; Tappertzhofen, S.; Waser, R.; Valov, I. Switching Kinetics of Electrochemical Metallization Memory Cells. Phys. Chem. Chem. Phys. 2013, 15, 6945-6952.

(12) Kozicki, M. N.; Mitkova, M.; Valov, I. Electrochemical Metallization Memories. In Resistive Switching: From Fundamentals of Nanoionic Redox Processes to Memristive Device Applications; 2016; pp. 483-514.

(13) Chung, A.; Deen, J.; Lee, J.-S.; Meyyappan, M.; Hutchby J A, B. G. I. Z. V. V. and B. J. E.; Brewer J E, Z. V. V. and H. J.; M, M.; Meyyappan M, S. M.; C, E. J. C. and L. J.; 


\section{WILEY-VCH}

Ong T-C, K. P. K. and H. C.; et al. Nanoscale Memory Devices. Nanotechnology 2010, 21,412001 .

(14) Bernard, Y.; Renard, V. T.; Gonon, P.; Jousseaume, V. Back-End-of-Line Compatible Conductive Bridging RAM Based on $\mathrm{Cu}$ and $\mathrm{SiO} 2$. Microelectron. Eng. 2011, 88, $814-816$.

(15) Belmonte, A.; Kim, W.; Chan, B.; Heylen, N.; Fantini, A.; Houssa, M.; Jurczak, M.;

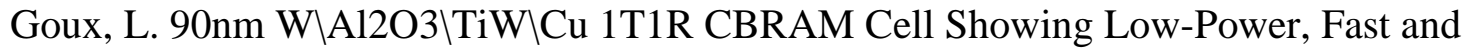
Disturb-Free Operation. In 2013 5th IEEE International Memory Workshop; IEEE, 2013; pp. 26-29.

(16) van den Hurk, J.; Valov, I.; Waser, R. Preparation and Characterization of GeSx ThinFilms for Resistive Switching Memories. Thin Solid Films 2013, 527, 299-302.

(17) Tappertzhofen, S.; Valov, I.; Waser, R. Quantum Conductance and Switching Kinetics of AgI-Based Microcrossbar Cells. Nanotechnology 2012, 23, 145703.

(18) Rajan, K.; Chiappone, A.; Perrone, D.; Bocchini, S.; Roppolo, I.; Bejtka, K.;

Castellino, M.; Pirri, C. F.; Ricciardi, C.; Chiolerio, A. Ionic Liquid-Enhanced Soft Resistive Switching Devices. RSC Adv. 2016, 6, 94128-94138.

(19) Rajan, K.; Bejtka, K.; Bocchini, S.; Perrone, D.; Chiappone, A.; Roppolo, I.; Pirri, C. F.; Ricciardi, C.; Chiolerio, A. Highly Performing Ionic Liquid Enriched Hybrid RSDs. J. Mater. Chem. C 2017, 5, 6144-6155.

(20) Tappertzhofen, S.; Valov, I.; Tsuruoka, T.; Hasegawa, T.; Waser, R.; Aono, M. Generic Relevance of Counter Charges for Cation-Based Nanoscale Resistive Switching Memories. ACS Nano 2013, 7, 6396-6402.

(21) Tsuruoka, T.; Terabe, K.; Hasegawa, T.; Valov, I.; Waser, R.; Aono, M. Effects of Moisture on the Switching Characteristics of Oxide-Based, Gapless-Type Atomic Switches. Adv. Funct. Mater. 2012, 22, 70-77.

(22) Messerschmitt, F.; Kubicek, M.; Rupp, J. L. M. How Does Moisture Affect the 


\section{WILEY-VCH}

Physical Property of Memristance for Anionic-Electronic Resistive Switching Memories? Adv. Funct. Mater. 2015, 25, 5117-5125.

(23) Tappertzhofen, S.; Menzel, S.; Valov, I.; Waser, R. Redox Processes in Silicon Dioxide Thin Films Using Copper Microelectrodes. Appl. Phys. Lett. 2011, 99, 203103.

(24) Tappertzhofen, S.; Mündelein, H.; Valov, I.; Waser, R. Nanoionic Transport and Electrochemical Reactions in Resistively Switching Silicon Dioxide. Nanoscale 2012, 4, 3040 .

(25) Tsuruoka, T.; Valov, I.; Tappertzhofen, S.; van den Hurk, J.; Hasegawa, T.; Waser, R.; Aono, M. Redox Reactions at Cu,Ag/Ta 2 O 5 Interfaces and the Effects of Ta 2 O 5 Film Density on the Forming Process in Atomic Switch Structures. Adv. Funct. Mater. 2015, 25, 6374-6381.

(26) Yang, Y.; Gao, P.; Li, L.; Pan, X.; Tappertzhofen, S.; Choi, S.; Waser, R.; Valov, I.; Lu, W. D. Electrochemical Dynamics of Nanoscale Metallic Inclusions in Dielectrics. Nat. Commun. 2014, 5, 4232.

(27) Tappertzhofen, S.; Hofmann, S. Embedded Nanoparticle Dynamics and Their Influence on Switching Behaviour of Resistive Memory Devices. Nanoscale 2017, 9, 1749417504.

(28) Menzel, S.; Böttger, U.; Wimmer, M.; Salinga, M. Physics of the Switching Kinetics in Resistive Memories. Adv. Funct. Mater. 2015, 25, 6306-6325.

(29) Wedig, A.; Luebben, M.; Cho, D.-Y.; Moors, M.; Skaja, K.; Rana, V.; Hasegawa, T.; Adepalli, K. K.; Yildiz, B.; Waser, R.; et al. Nanoscale Cation Motion in TaOx, HfOx and TiOx Memristive Systems. Nat. Nanotechnol. 2015, 11, 67-74.

(30) Lübben, M.; Wiefels, S.; Waser, R.; Valov, I. Processes and Effects of Oxygen and Moisture in Resistively Switching TaOx and HfOx. Adv. Electron. Mater. 2018, 4.

(31) Kim, Y.-M.; Lee, J.-S. Reproducible Resistance Switching Characteristics of Hafnium Oxide-Based Nonvolatile Memory Devices. J. Appl. Phys. 2008, 104, 114115. 


\section{WILEY-VCH}

(32) Chen, Y.-S.; Lee, H.-Y.; Chen, P.-S.; Liu, W.-H.; Wang, S.-M.; Gu, P.-Y.; Hsu, Y.-Y.; Tsai, C.-H.; Chen, W.-S.; Chen, F.; et al. Robust High-Resistance State and Improved Endurance of $\langle$ formula Formulatype="inline" $>\langle$ tex Notation="TeX" $>$ \$lhbox $\{\text { HfO }\}_{-}\{\mathrm{X}\} \$</$ Tex $></$ Formula $>$ Resistive Memory by Suppression of Current Overshoot. IEEE Electron Device Lett. 2011, 32, 1585-1587.

(33) Hou, Y.; Celano, U.; Goux, L.; Liu, L.; Degraeve, R.; Cheng, Y.; Kang, J.; Jurczak, M.; Vandervorst, W. Multimode Resistive Switching in Nanoscale Hafnium Oxide Stack as Studied by Atomic Force Microscopy. Appl. Phys. Lett. 2016, 109, 023508.

(34) Müller, J.; Böscke, T. S.; Schröder, U.; Mueller, S.; Bräuhaus, D.; Böttger, U.; Frey, L.; Mikolajick, T. Ferroelectricity in Simple Binary $\mathrm{ZrO}_{2}$ and $\mathrm{HfO}_{2}$. Nano Lett. 2012, $12,4318-4323$.

(35) Max, B.; Pešić, M.; Slesazeck, S.; Mikolajick, T. Interplay between Ferroelectric and Resistive Switching in Doped Crystalline $\mathrm{HfO}_{2}$. J. Appl. Phys. 2018, 123, 134102.

(36) Chiolerio, A.; Roppolo, I.; Bejtka, K.; Asvarov, A.; Pirri, C. F. Resistive Hysteresis in Flexible Nanocomposites and Colloidal Suspensions: Interfacial Coupling Mechanism Unveiled. $R S C A d v$. 2016, 6, 56661-56667.

(37) Wagenaar, J. J. T.; Morales-Masis, M.; van Ruitenbeek, J. M. Observing "Quantized" Conductance Steps in Silver Sulfide: Two Parallel Resistive Switching Mechanisms. J. Appl. Phys. 2012, 111, 014302.

(38) Syu, Y.-E.; Chang, T.-C.; Lou, J.-H.; Tsai, T.-M.; Chang, K.-C.; Tsai, M.-J.; Wang, Y.L.; Liu, M.; Sze, S. M. Atomic-Level Quantized Reaction of HfOx Memristor. Appl. Phys. Lett. 2013, 102, 172903.

(39) Sun, B.; Liu, L.; Xu, N.; Gao, B.; Wang, Y.; Han, D.; Liu, X.; Han, R.; Kang, J. The Effect of Current Compliance on the Resistive Switching Behaviors in TiN/ZrO 2 /Pt Memory Device. Jpn. J. Appl. Phys. 2009, 48, 04 C061.

(40) Krishnan, K.; Aono, M.; Tsuruoka, T. Kinetic Factors Determining Conducting 


\section{WILEY-VCH}

Filament Formation in Solid Polymer Electrolyte Based Planar Devices. Nanoscale 2016.

(41) Rutman, J.; Riess, I. Reference Electrodes for Thin-Film Solid-State Ionic Devices. Solid State Ionics 2008, 179, 108-112.

(42) Lübben, M.; Karakolis, P.; Ioannou-Sougleridis, V.; Normand, P.; Dimitrakis, P.; Valov, I. Graphene-Modified Interface Controls Transition from VCM to ECM Switching Modes in Ta/TaO x Based Memristive Devices. Adv. Mater. 2015, 27, 6202-6207.

(43) Hubbard, W. A.; Kerelsky, A.; Jasmin, G.; White, E. R.; Lodico, J.; Mecklenburg, M.; Regan, B. C. Nanofilament Formation and Regeneration During Cu/Al 2 O 3 Resistive Memory Switching. Nano Lett. 2015, 15, 3983-3987.

(44) D’Aquila, K.; Liu, Y.; Iddir, H.; Petford-Long, A. K. In Situ TEM Study of Reversible and Irreversible Electroforming in Pt/Ti:NiO/Pt Heterostructures. Phys. status solidi Rapid Res. Lett. 2015, 9, 301-306.

(45) Yang, Y.; Lu, W. D. Progress in the Characterizations and Understanding of Conducting Filaments in Resistive Switching Devices. IEEE Trans. Nanotechnol. 2016, $P P, 1-1$.

(46) Chiolerio, A.; Perrone, D.; Roppolo, I.; Rizza, G.; Risplendi, F.; Stassi, S.; Laurenti, M.; Rajan, K.; Chiappone, A.; Bocchini, S.; et al. Nanoparticle Reshaping and Ion Migration in Nanocomposite Ultrafast Ionic Actuators: The Converse Piezo-ElectroKinetic Effect. Adv. Funct. Mater. 2019, 29, 1902941.

(47) Kwon, D.-H.; Kim, K. M.; Jang, J. H.; Jeon, J. M.; Lee, M. H.; Kim, G. H.; Li, X.-S.; Park, G.-S.; Lee, B.; Han, S.; et al. Atomic Structure of Conducting Nanofilaments in TiO2 Resistive Switching Memory. Nat. Nanotechnol. 2010, 5, 148-153.

(48) Peppler, K.; Reitz, C.; Janek, J. Field-Driven Migration of Bipolar Metal Particles on Solid Electrolytes. Appl. Phys. Lett. 2008, 93, 074104. 


\section{WILEY-VCH}

(49) Mayer, J.; Giannuzzi, L. A.; Kamino, T.; Michael, J. TEM Sample Preparation and FIB-Induced Damage. MRS Bull. 2011, 32, 400-407.

(50) Di Martino, G.; Tappertzhofen, S.; Hofmann, S.; Baumberg, J. Nanoscale PlasmonEnhanced Spectroscopy in Memristive Switches. Small 2016, 12, 1334-1341.

(51) Kos, D.; Astier, H. P. A. G.; Di Martino, G.; Mertens, J.; Ohadi, H.; De Fazio, D.; Yoon, D.; Zhao, Z.; Kuhn, A.; Ferrari, A. C.; et al. Electrically Controlled Nano and Micro Actuation in Memristive Switching Devices with On-Chip Gas Encapsulation. Small 2018, 14, 1801599.

(52) Bohren, C. F.; Huffman, D. R. Absorption and Scattering of Light by Small Particles; Wiley-VCH Verlag: Weinheim, Germany, 1983.

(53) Hao, E.; Schatz, G. C. Electromagnetic Fields around Silver Nanoparticles and Dimers. J. Chem. Phys. 2003, 120, 357-366.

(54) Savage, K. J.; Hawkeye, M. M.; Esteban, R.; Borisov, A. G.; Aizpurua, J.; Baumberg, J. J. Revealing the Quantum Regime in Tunnelling Plasmonics. Nature 2012, 491, $574-577$.

(55) Taylor, R. W.; Esteban, R.; Mahajan, S.; Aizpurua, J.; Baumberg, J. J. Optimizing SERS from Gold Nanoparticle Clusters: Addressing the Near Field by an Embedded Chain Plasmon Model. J. Phys. Chem. C 2016, 120, 10512-10522.

(56) Myroshnychenko, V.; Rodríguez-Fernández, J.; Pastoriza-Santos, I.; Funston, A. M.; Novo, C.; Mulvaney, P.; Liz-Marzán, L. M.; García de Abajo, F. J. Modelling the Optical Response of Gold Nanoparticles. Chem. Soc. Rev. 2008, 37, 1792.

(57) Lévêque, G.; Martin, O. J. F. Optical Interactions in a Plasmonic Particle Coupled to a Metallic Film. Opt. Express 2006, 14, 9971.

(58) Benz, F.; de Nijs, B.; Tserkezis, C.; Chikkaraddy, R.; Sigle, D. O.; Pukenas, L.; Evans, S. D.; Aizpurua, J.; Baumberg, J. J. Generalized Circuit Model for Coupled Plasmonic Systems. Opt. Express 2015, 23, 33255. 


\section{WILEY-VCH}

(59) Emboras, A.; Niegemann, J.; Ma, P.; Haffner, C.; Pedersen, A.; Luisier, M.; Hafner, C.; Schimmel, T.; Leuthold, J. Atomic Scale Plasmonic Switch. Nano Lett. 2016, 16, 709714.

(60) Emboras, A.; Goykhman, I.; Desiatov, B.; Mazurski, N.; Stern, L.; Shappir, J.; Levy, U. Nanoscale Plasmonic Memristor with Optical Readout Functionality. 2013.

(61) Emboras, A.; Alabastri, A.; Ducry, F.; Cheng, B.; Salamin, Y.; Ma, P.; Andermatt, S.; Baeuerle, B.; Josten, A.; Hafner, C.; et al. Atomic Scale Photodetection Enabled by a Memristive Junction. ACS Nano 2018, 12, 6706-6713.

(62) Lin, H.; Ogbuu, O.; Liu, J.; Zhang, L.; Michel, J.; Hu, J. Breaking the EnergyBandwidth Limit of Electrooptic Modulators: Theory and a Device Proposal. J. Light. Technol. 2013, 31, 4029-4036.

(63) Sun, C.; Wade, M. T.; Lee, Y.; Orcutt, J. S.; Alloatti, L.; Georgas, M. S.; Waterman, A. S.; Shainline, J. M.; Avizienis, R. R.; Lin, S.; et al. Single-Chip Microprocessor That Communicates Directly Using Light. Nature 2015, 528, 534-538. 